\title{
Charm quark system on the physical point in $2+1$ flavor lattice QCD
}

\section{Y. Namekawa* 'for PACS-CS Collaboration,}

Center for Computational Sciences, University of Tsukuba, Tsukuba, Ibaraki 305-8577, Japan

\begin{abstract}
We investigate the charm quark system on $2+1$ flavor PACS-CS configurations. Calculations are performed at the lattice spacing $a^{-1}=2.194(10) \mathrm{GeV}$ and the spatial extent $L=2.9 \mathrm{fm}$ with O(a)improved Wilson fermions for the light quarks and the relativistic heavy fermion for the charm quark. Our dynamical $u d$ and strange quark masses and valence charm quark mass are set to their physical values. A comparison of the mass spectrum and decay constants with experiments is presented. Our results for the charm quark mass and CKM matrix elements are also reported.
\end{abstract}

The XXIX International Symposium on Lattice Field Theory - Lattice 2011

July 10-16, 2011

Squaw Valley, Lake Tahoe, California

\footnotetext{
*Speaker.

${ }^{\dagger}$ E-mail: namekawa@ccs.tsukuba.ac.jp
} 


\section{Introduction}

Precise determination of the Cabibbo-Kobayashi-Maskawa (CKM) quark mixing matrix is an indispensable step to establish the validity range of the standard model, and to search for new physics at higher energy scales. Lattice QCD has been making steady progress in this direction.

One of the difficulties with the charm quark in lattice QCD simulations at a typical cutoff $a^{-1} \approx 2 \mathrm{GeV}$ resides in significant cutoff errors due to the charm quark mass. The heavy quark mass correction is $m_{Q} a \sim O(1)$, and hence it is desirable to control $m_{Q} a$ errors to achieve an accuracy of a few percent. The Fermilab action [ [W] and the relativistic heavy quark action [0, [3] have been proposed to meet this goal. The relativistic heavy quark action removes the leading cutoff errors of $O\left(\left(m_{Q} a\right)^{n}\right)$ and the next to leading effects of $O\left(\left(m_{Q} a\right)^{n}\left(a \Lambda_{Q C D}\right)\right)$ with arbitrary order $n$, once all of the parameters in the heavy quark action are determined nonperturbatively.

Another source that prevents precise evaluations in lattice QCD is the error associated with chiral extrapolations in the light quark masses. This problem has been increasingly alleviated through progress toward simulations with lighter and lighter dynamical quark masses. The acceleration of dynamical lattice QCD simulation using multi-time steps for infrared and ultraviolet modes has made it possible to run simulations with light up, down, and strange quark masses around their physical values [䧃]. In fact, we can proceed one more step and reweight dynamical simulations such that dynamical quark masses take exactly the physical values [[]]. Once the reweighting is successfully made, ambiguities associated with chiral extrapolations are completely removed.

In this paper, we present our work for the charm quark system treated with the relativistic heavy quark formalism on the $2+1$ dynamical flavor PACS-CS configurations of $32^{3} \times 64$ lattice generated with the Wilson-clover quark and reweighted to the physical point for up, down and strange quark masses. Details of our calculations are presented in Ref. [G].

\section{Set up}

Our calculation is based on a set of $N_{f}=2+1$ flavor dynamical lattice QCD configurations generated by the PACS-CS Collaboration [D] on a $32^{3} \times 64$ lattice using the nonperturbatively $O(a)$-improved Wilson quark action with $c_{\mathrm{SW}}^{\mathrm{NP}}=1.715[\mathrm{U}]$ and the Iwasaki gauge action at $\beta=$ 1.90. The aggregate of $2000 \mathrm{MD}$ time units were generated at the hopping parameter given by $\left(\kappa_{u d}^{0}, \kappa_{s}^{0}\right)=(0.13778500,0.13660000)$, and 80 configurations at every $25 \mathrm{MD}$ time units were used for measurements. We then reweight those configurations to the physical point given by $\left(\kappa_{u d}, \kappa_{s}\right)=(0.13779625,0.13663375)$. The reweighting shifts the masses of $\pi$ and $K$ mesons from $m_{\pi}=152(6) \mathrm{MeV}$ and $m_{K}=509(2) \mathrm{MeV}$ to $m_{\pi}=135(6) \mathrm{MeV}$ and $m_{K}=498(2) \mathrm{MeV}$, with the cutoff at the physical point estimated to be $a^{-1}=2.194(10) \mathrm{GeV}$.

Our relativistic heavy quark action [D] is given by

$$
\begin{aligned}
S_{Q}= & \sum_{x, y} \bar{Q}_{x} D_{x, y} Q_{y} \\
D_{x, y}= & \delta_{x y}-\kappa_{Q} \sum_{i}\left[\left(r_{s}-v \gamma_{i}\right) U_{x, i} \delta_{x+\hat{i}, y}+\left(r_{s}+v \gamma_{i}\right) U_{x, i}^{\dagger} \delta_{x, y+\hat{i}}\right] \\
& -\kappa_{Q}\left[\left(r_{t}-\gamma_{4}\right) U_{x, 4} \delta_{x+\hat{4}, y}+\left(r_{t}+\gamma_{4}\right) U_{x, 4}^{\dagger} \delta_{x, y+\hat{4}}\right]
\end{aligned}
$$


Table 1: Simulation parameters. MD time is the number of trajectories multiplied by the trajectory length.

\begin{tabular}{ccccc}
\hline$\beta$ & $\kappa_{\text {ud }}$ & $\kappa_{\mathrm{s}}$ & $\#$ conf & MD time \\
\hline 1.90 & 0.13779625 & 0.13663375 & 80 & 2000 \\
\hline
\end{tabular}

Table 2: Parameters for the relativistic heavy quark action.

\begin{tabular}{ccccc}
\hline$\kappa_{\text {charm }}$ & $v$ & $r_{s}$ & $c_{B}$ & $c_{E}$ \\
\hline 0.10959947 & 1.1450511 & 1.1881607 & 1.9849139 & 1.7819512 \\
\hline & $-\kappa_{Q}\left[c_{B} \sum_{i, j} F_{i j}(x) \sigma_{i j}+c_{E} \sum_{i} F_{i 4}(x) \sigma_{i 4}\right] \delta_{x y}$,
\end{tabular}

where $\kappa_{Q}$ is the hopping parameter for the heavy quark. The parameters $r_{t}, r_{s}, c_{B}, c_{E}$, and $v$ are adjusted as follows. We are allowed to choose $r_{t}=1$, and we employ a one-loop perturbative value for $r_{s}\left[[]\right.$. For the clover coefficients $c_{B}$ and $c_{E}$, we include the non-perturbative contribution in the massless limit $c_{\mathrm{SW}}^{\mathrm{NP}}$ for three flavor dynamical QCD [प], and calculate the heavy quark mass dependent contribution to one-loop order in perturbation theory [ [ $]$ ]. The parameter $v$ is determined non-perturbatively to reproduce the relativistic dispersion relation for the spin-averaged $1 S$ states of the charmonium. Writing

$$
E(\vec{p})^{2}=E(\overrightarrow{0})^{2}+c_{\mathrm{eff}}^{2}|\vec{p}|^{2},
$$

for $|\vec{p}|=0,(2 \pi / L), \sqrt{2}(2 \pi / L)$, and demanding the effective speed of light $c_{\mathrm{eff}}$ to be unity, we find $v=1.1450511$ with which we have $c_{\text {eff }}=1.002(4)$. It is noted that the cutoff error of $O\left(\alpha_{s}^{2}\left(a \Lambda_{Q C D}\right)\right)$ remains, in addition to $O\left(\left(a \Lambda_{Q C D}\right)^{2}\right)$, due to the use of one-loop perturbative values in part for the parameters of our heavy quark action.

We tune the heavy quark hopping parameter to reproduce an experimental value of the mass for the spin-averaged $1 S$ states of the charmonium, given by $M(1 S)^{\exp }=\left(M_{\eta_{c}}+3 M_{J / \psi}\right) / 4=$ $3.0678(3) \mathrm{GeV}$ [Q] . This leads to $\kappa_{\text {charm }}=0.10959947$ for which our lattice QCD measurement yields the value $M(1 S)^{l a t}=3.067(1)(14) \mathrm{GeV}$, where the first error is statistical, and the second is a systematic from the scale determination. Our parameters for the relativistic heavy quark action are summarized in Table [.

Statistical errors are analyzed by the jackknife method with a bin size of 100 MD time units (4 configurations), as in the light quark sector [5].

\section{Charmonium spectrum and charm quark mass}

Our results for the charmonium spectrum on the physical point are summarized in Fig. $\mathrm{W}$. Within the error of $0.5-1 \%$, the predicted spectrum is in reasonable agreement with experiment.

Let us consider the $1 S$ states more closely. Since these states are employed to tune the charm quark mass, the central issue here is the magnitude of the hyperfine splitting. Our result $m_{J / \psi}-$ $m_{\eta_{c}}=0.108(1)(0) \mathrm{GeV}$, where the first error is statistical and the second error is systematic from 

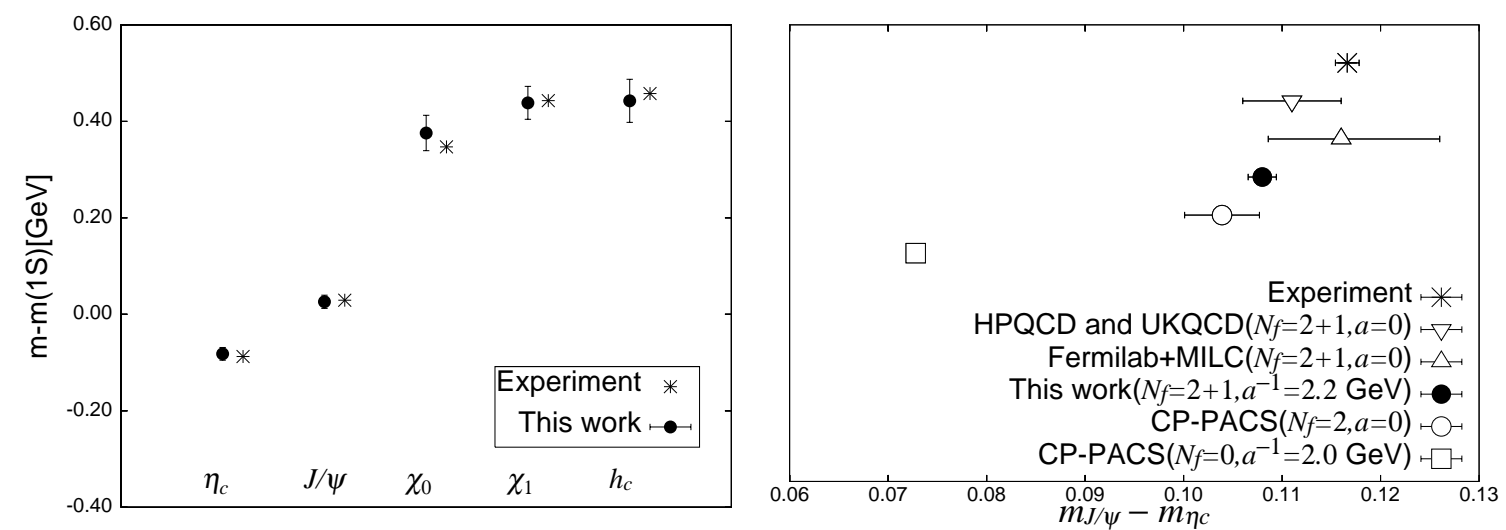

Figure 1: Our results for the charmonium mass spectrum(left panel) and the hyperfine splitting of the charmonium with different number of flavors(right panel).

the scale determination, is $7 \%$ smaller than the experimental value of $0.117 \mathrm{GeV}$. In Fig. 四, we compare the present result on $N_{f}=2+1$ flavor dynamical configurations with previous attempts on $N_{f}=2$ dynamical and quenched configurations using the same heavy quark formalism and the Iwasaki gluon action [ए]]. Other results by recent lattice QCD simulations by Fermilab lattice and MILC Collaborations []], HPQCD and UKQCD Collaborations [ㅁ] ] are also plotted. We observe a clear trend that incorporation of dynamical light quark effects improves the agreement.

We should note that we have not evaluated our systematic errors for the hyperfine splitting, yet. The continuum extrapolation needs to be performed. A naive order counting implies that the cutoff effects of $O\left(\alpha_{s}^{2}\left(a \Lambda_{Q C D}\right),\left(a \Lambda_{Q C D}\right)^{2}\right)$ from the relativistic heavy quark action are at a percent level. Another aspect is that dynamical charm quark effects and disconnected loop contributions, albeit reported to give a shift of only a few $\mathrm{MeV}$ [[3], are not included in the present work. Additional calculations are needed to draw a definite conclusion for the hyperfine splitting of the charmonium spectrum. We leave it for a future work.

Using the axial vector Ward-Takahashi identity, the charm quark mass is obtained. The systematic error due to the heavy quark of $O\left(\alpha_{s}^{2}\left(a \Lambda_{Q C D}\right),\left(a \Lambda_{Q C D}\right)^{2}\right)$ will be estimated by data on finer lattices in the future. Figure $\square$ compares our result with a recent $N_{f}=2+1$ lattice QCD estimation by the HPQCD Collaboration [ए2]. Another result by ETM Collaboration is also plotted [14]]. In addition to lattice QCD determinations, recent continuum results using the Heavy Quark Expansions(HQE) [ए5], as well as sum rules [ए6], are shown. All these results are consistent.

\section{Charmed meson and charmed-strange meson spectrum}

We calculate the charmed meson and charmed-strange meson masses which are stable on our lattice with the spatial size of $L=2.88(1) \mathrm{fm}$ and a lattice cutoff of $a^{-1}=2.194(10) \mathrm{GeV}$. The $D^{*}$ and $D_{s}^{*}$ meson decay channels are not open in our lattice setup. $D_{s 0}^{*}$ and $D_{s 1}$ meson masses are below the $D K$ threshold [ $\left[\right.$ ] but above the $D_{s} \pi$ threshold. Their decays, however, are prohibited by the isospin symmetry. On the other hand, $D_{0}^{*}$ and $D_{1}$ meson masses are not computed since their decay channels are open, and therefore a calculation involving $D \pi$ contributions is needed. 


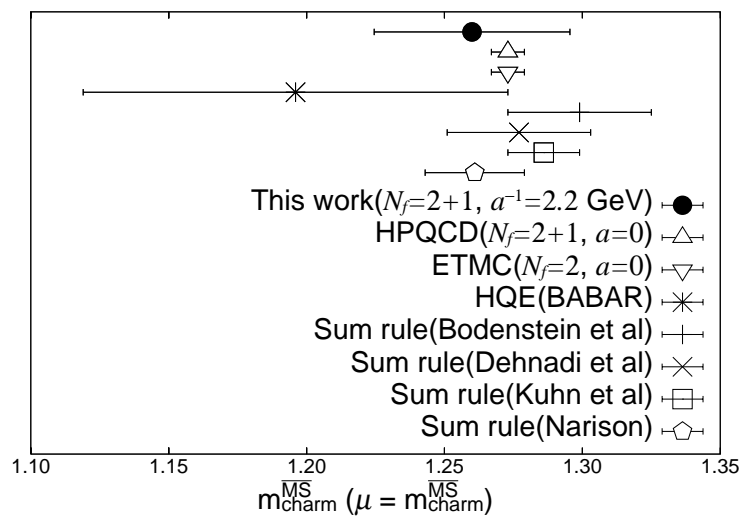

Figure 2: Comparison of the charm quark mass. We employ $N_{f}=4$ running in this plot.
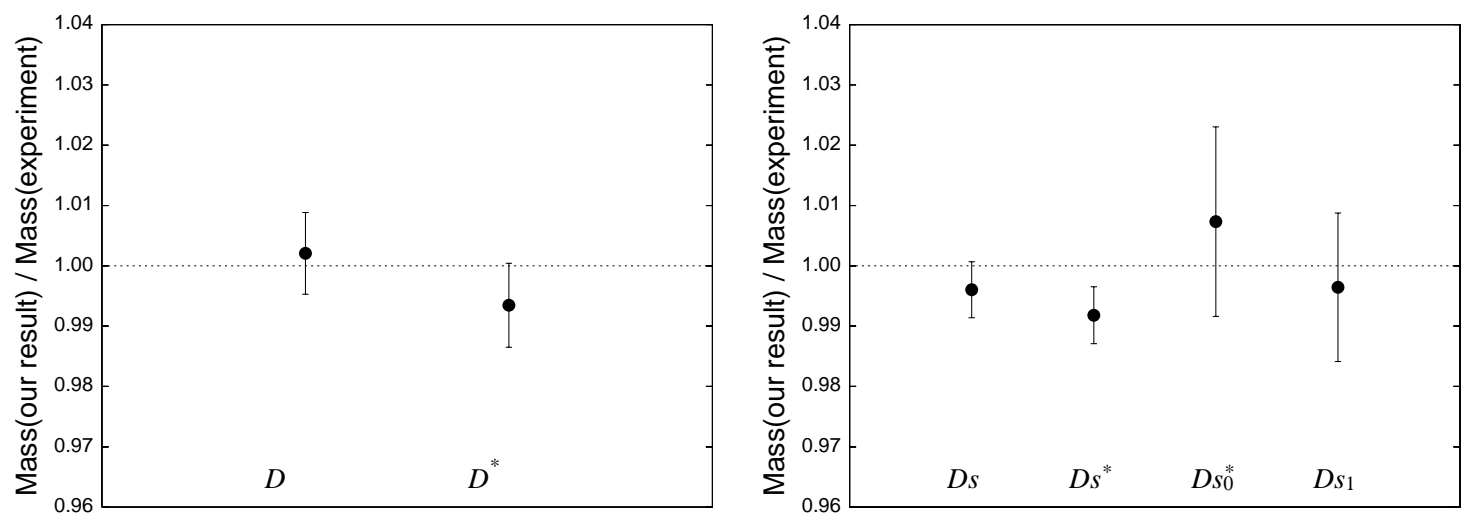

Figure 3: Our results for charmed meson masses(left panel) and charmed-strange meson masses(right panel) normalized by the experimental values.

Our results are summarized in Fig. [3]. All our values for the heavy-light meson quantities are predictions, because the physical charm quark mass has already been fixed with the charmonium spectrum. The experimental spectrum are reproduced in $2 \sigma$ level. The potential model predicts the $D_{s 0}^{*}$ meson mass is above the $D K$ threshold [ㅁ]], which deviates from the experiment significantly [[8]. Our result, however, does not indicate such a large difference from the experimental value. A similar result is obtained in other lattice QCD calculations [ [प्Q, 20]. The $D_{s 0}^{*}$ meson mass is below the $D K$ threshold. It should be noticed that our calculation does not cover $D K$ scattering states yet. A $D K$ contamination for $D_{s 0}^{*}$ and $D_{s 1}$ meson masses could be considerably large. Further analysis is required to validate our results for $D_{s 0}^{*}$ and $D_{s 1}$ meson spectrum.

Figure 6 shows our decay constants including the experimental values [Q], as well as three recent lattice QCD results: HPQCD and UKQCD Collaboration [12], Fermilab lattice and MILC

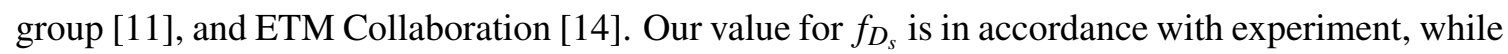
that for $f_{D}$ is somewhat larger. Comparing four sets of lattice determinations, we observe, both for $f_{D}$ and $f_{D_{s}}$, an agreement between our values and those of the Fermilab group, while there seems to be a discrepancy between our values and those by the HPQCD and UKQCD Collaboration and 

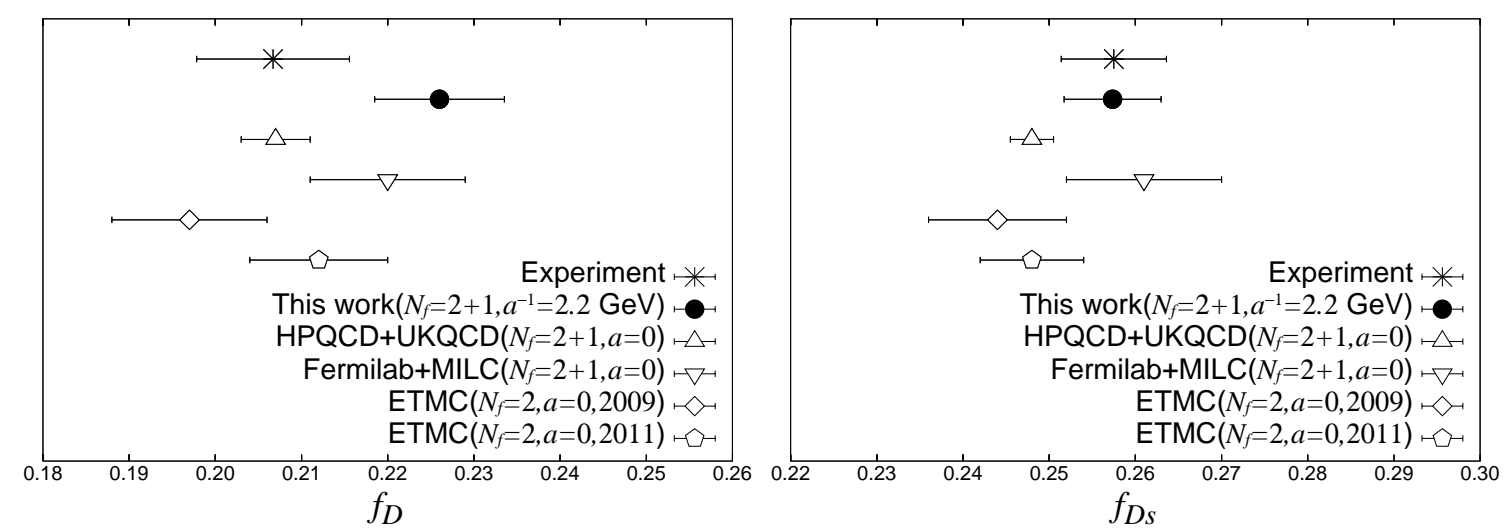

Figure 4: Comparison of pseudoscalar decay constants for the charmed meson (left panel) and charmedstrange meson (right panel).
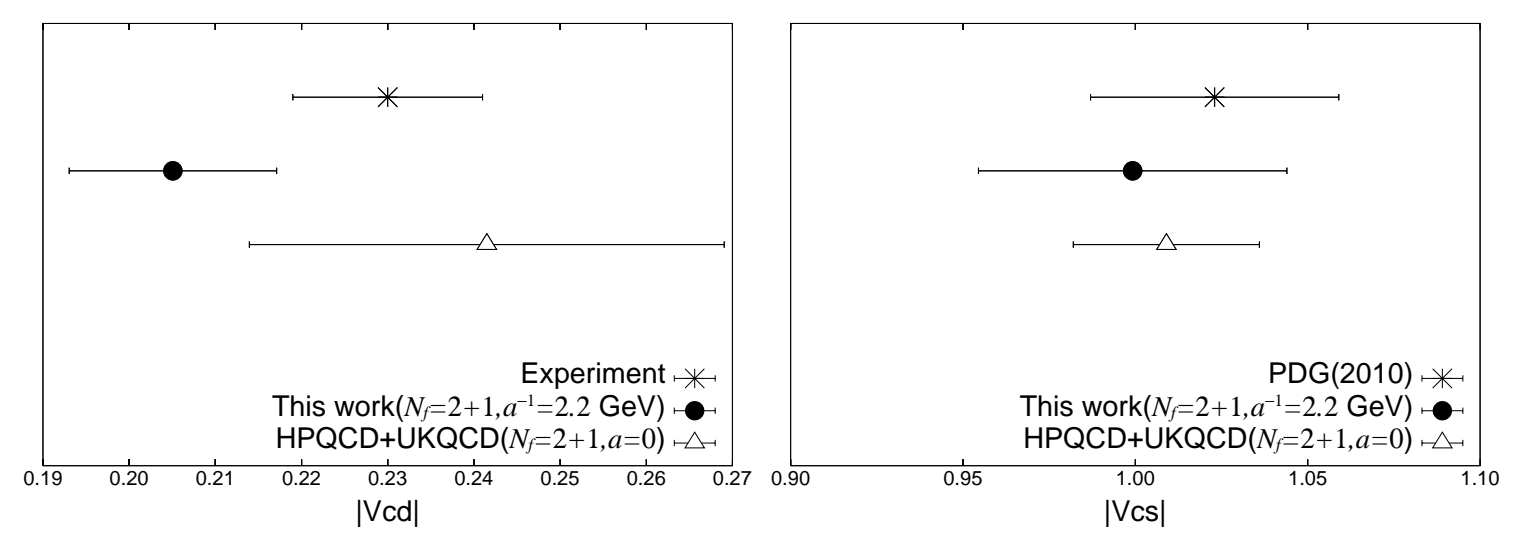

Figure 5: Comparison of the CKM matrix elements, $\left|V_{c d}\right|$ (left panel) and $\left|V_{c s}\right|$ (right panel).

ETM Collaboration, though continuum extrapolation is needed on our part.

The standard model relates $\left|V_{c d}\right|$ to the leptonic decay width of the $D$ meson $\Gamma(D \rightarrow l v)$ by

$$
\Gamma(D \rightarrow l v)=\frac{G_{F}^{2}}{8 \pi} f_{D}^{2} m_{l}^{2} m_{D}\left(1-\frac{m_{l}^{2}}{m_{D}^{2}}\right)^{2}\left|V_{c d}\right|^{2},
$$

where $G_{F}$ is the Fermi coupling constant, and $m_{l}$ is the lepton mass in the final state. A lattice determination of the $D$ meson decay constant $f_{D}$ with the experimental value of $\Gamma(D \rightarrow l v)$ gives $\left|V_{c d}\right| .\left|V_{c s}\right|$ can be obtained in the same way.

We estimate $\left|V_{c d}\right|$ from our $D$ meson mass and decay constant with the CLEO value of $\Gamma(D \rightarrow$ $l v)$ []] . Up to our heavy quark discretization error of $O\left(\alpha_{s}^{2}\left(a \Lambda_{Q C D}\right),\left(a \Lambda_{Q C D}\right)^{2}\right)$, we obtain $\left|V_{c d}\right|$. Our result of $\left|V_{c d}\right|$ is about $10 \%$ smaller than the PDG value [Q] as shown in Fig. [1. Similarly, using the CLEO value of $\Gamma\left(D_{s} \rightarrow l v\right)$ [2]], we find $\left|V_{c s}\right|$, which is consistent with PDG.

\section{Acknowledgments}

Y.N. thanks Takayuki Matsuki for helpful comments. Numerical calculations for the present 
work have been carried out on the PACS-CS computer under the "Interdisciplinary Computational Science Program" of the Center for Computational Sciences, University of Tsukuba. This work is supported in part by Grants-in-Aid of the Ministry of Education, Culture, Sports, Science and Technology-Japan (Nos. 18104005, 20340047, 20540248, 21340049, 22244018, and 22740138), and Grant-in-Aid for Scientific Research on Innovative Areas (No. 2004: 20105001, 20105002, 20105003, 20105005, and No. 2104: 22105501)

\section{References}

[1] A.X. El-Khadra et al., Phys. Rev. D 55, 3933 (1997); M.B. Oktay and A.S. Kronfeld, Phys. Rev. D 78, 014504 (2008).

[2] S. Aoki et al., Prog. Theor. Phys. 109, 383 (2003).

[3] N.H. Christ et al., Phys. Rev. D 76, 074505 (2007).

[4] S. Aoki et al. (PACS-CS Collaboration), Phys. Rev. D 79, 034503 (2009).

[5] S. Aoki et al. (PACS-CS Collaboration), Phys. Rev. D 81, 074503 (2010).

[6] Y. Namekawa et al. (PACS-CS Collaboration), arXiv:1104.4600.

[7] S. Aoki et al. (CP-PACS and JLQCD Collaborations), Phys. Rev. D 73, 034501 (2006).

[8] S. Aoki et al., Nucl. Phys. B 697, 271 (2004).

[9] K. Nakamura et al. (Particle Data Group), J. Phys. G 37, 075021 (2010)

[10] Y. Kayaba et al. (CP-PACS Collaboration), JHEP 0702, 019 (2007); Y. Kayaba, Ph.D. thesis, University of Tsukuba, 2005.

[11] T. Burch et al. (Fermilab Lattice and MILC Collaboration), Phys. Rev. D 81, 034508 (2010); J.A. Bailey et al. (Fermilab Lattice and MILC Collaboration), PoS(LATTICE 2010), 317 (2010).

[12] E. Follana et al. (HPQCD and UKQCD Collaboration), Phys. Rev. D 75, 054502 (2007); Phys. Rev. Lett. 100062002 (2008); C. McNeile et al. (HPQCD Collaboration), Phys. Rev. D 82, 034512 (2010); C.T.H. Davies et al., Phys. Rev. D 82, 114504 (2010).

[13] C. McNeile et al. (UKQCD Collaboration), Phys. Rev. D 70, 034506 (2004); P. de Forcrand et al. (QCD-TARO Collaboration), JHEP 0408, 004 (2004); C. Ehmann and G.S. Bali, PoS(LATTICE 2008), 114 (2008); L. Levkova and C.E. DeTar, Phys. Rev. D 83, 074504 (2011).

[14] B. Blossier et al. (European Twisted Mass Collaboration), JHEP 0907, 043 (2009); ibid, Phys. Rev. D 82, 114513 (2010); P. Dimopoulos et al. (European Twisted Mass Collaboration), arXiv:1107.1441.

[15] B. Aubert et al. (BABAR Collaboration), Phys. Rev. D 81, 032003 (2010).

[16] J.H. Kuhn et al., Nucl. Phys. B 778, 192 (2007); S. Bodenstein et al., Phys. Rev. D 82, 114013 (2010); S. Narison, Phys. Lett. B 693559 (2010); B. Dehnadi et al., arXiv:1102.2264.

[17] S. Godfrey and N. Isgur, Phys. Rev. D 32, 189 (1985).

[18] For reviews, E.S. Swanson, Phys. Rep. 429, 243 (2006); S.L. Zhu, Int. J. Mod. Phys. E 17, 283 (2008).

[19] S.J. Dong et al. ( $\chi$ QCD Collaboration), PoS(LATTICE 2009), 090 (2009); M. Gong et al., PoS(LATTICE 2010), 106 (2010).

[20] D. Mohler and R.M. Woloshyn, Phys. Rev. D 84, 054505 (2011); PoS(LATTICE 2011), 130 (2011).

[21] J.P. Alexander et al. (CLEO Collaboration), Phys. Rev. D 79, 052001 (2009); B.I. Eisenstein et al. (CLEO Collaboration), Phys. Rev. D 78, 052003 (2008). 